\title{
Minimally invasive epicardial posterior left atrial wall isolation: technique and results
}

\author{
Mariana Floria ${ }^{*, 1,2}$, Mihaela Grecu ${ }^{3}$, Grigore Tinică ${ }^{2,3}$ \\ ${ }^{1} 3^{\text {rd }}$ Internal Medicine Department, "Sf. Spiridon" University Emergency Hospital lasi, Romania; \\ 2"Grigore T. Popa" University of Medicine and Pharmacy lasi, Romania; "3"Prof. Dr. George I.M. \\ Georgescu" Institute of Cardiovascular Diseases, lasi, Romania
}

\begin{abstract}
Atrial fibrillation (AF) is the most frequent arrhythmia in clinical practice. Catheter and surgical ablation has emerged as an alternative to maintain sinus rhythm and to avoid long-term AF complications. An ablation technique aims to target the triggers and the substrate of AF to prevent initiation and perpetuation of this arrhythmia. Surgical ablation is the gold standard in AF ablation; it has the best results in maintaining sinus rhythm in patients with persistent AF. Epicardial posterior left atrial wall isolation by right monolateral thoracoscopic approach is a minim invasive surgical technique that aims to eliminate major ganglionated plexi isolation influence on atrial myocardium in addition to pulmonary vein isolation. The exclusion of the left atrial posterior wall, including the pulmonary veins (considered as triggers or initiators) could be completed with an additional isolation/destruction of the adjacent major ganglionated plexi (considered as substrate modifiers) including the complex interconnection neural network which could add to influence the persistence of AF. Different hybrid surgical ablation lesions sets were developed, usually in a manner less than the full Cox-Maze IV lesion set. They are performed epicardially via minimally invasive (non-sternotomy) approaches without cardiopulmonary bypass, followed by catheter-based endocardial mapping, and if necessary additional ablation lines. The results of these innovative techniques are promising in persistent and long persistent AF. Epicardial right monolateral thoracoscopic approach to isolate the pulmonary vein and the major ganglionated plexi isolation in symptomatic refractory persistent AF patients is feasible and efficient.
\end{abstract}

Keywords: surgical ablation, epicardial ablation, refractory persistent atrial fibrillation, minimally invasive

\section{Introduction}

More than $30 \%$ of patients with arrhythmias at hospital admission have atrial fibrillation (AF) [1]. Persistent AF is defined as recurrent $A F$ that is sustained for seven days. In addition, patients with continuous AF who

Received: May 2017; Accepted after review: June 2017; Published: June 2017.

${ }^{*}$ Corresponding author: Mariana Floria, "Sf. Spiridon" University Emergency Hospital; "Grigore T. Popa" University of Medicine and Pharmacy, 16 University Street, 700115, lasi, Romania

E-mail: floria mariana@yahoo.com undergo cardioversion within seven days should be classified as having paroxysmal AF if the cardioversion is performed within 48 hours of AF onset, and persistent AF if the cardioversion is performed more than 48 hours after AF onset. Longstanding persistent $A F$ is defined as continuous AF of greater than one year's duration [2].

A major evolution has been made in the treatment of AF during the last years, offering now the potential to influence the outcome in a substantial number of patients by ablation. Despite this, treatment of persistent or longstanding persistent $\mathrm{AF}$ is a continuing challenge for every cardiologist. In patients who have not responded to antiarrhythmic drugs, the use of ablation (catheter and 
surgical) has emerged as an alternative to maintain sinus rhythm to avoid long-term AF complications. It is known that paroxysmal AF ablation is associated with a $35 \%$ and $66 \%$ larger probability of success as compared to ablation of persistent and longstanding persistent AF, respectively [3]. Surgical ablation seems to be the most efficient treatment in these patients with persistent $\mathrm{AF}$ and therefore should be taking into account as an attractive alternative.

According to the current guidelines an AF surgical ablation procedure is reasonable for selected patients with AF undergoing cardiac surgery for other indications (class Ila indication; level of evidence: C) [2]. For patients with AF undergoing cardiac surgery, there is moderate-quality evidence that concomitant AF surgery approximately doubles the percentage of freedom from $A F$, atrial flutter, or atrial tachycardia off antiarrhythmic drugs while increasing the risk of permanent pacemaker implantation [4]. However, due to the need for open-heart surgery and the morbidity associated with the surgical Cox-Maze procedure, minimally invasive and epicardial-endocardial (hybrid) ablation procedures have been developed. A stand-alone AF surgical ablation procedure (when additional cardiac surgical procedures are not performed) may be reasonable for selected patients with highly symptomatic AF not well managed with other approaches (class Ilb indication; level of evidence: B) [2]. A stand-alone procedure of surgical ablation must be guided by the following principles: a left atrial procedure should consist of pulmonary vein ablation, ideally with a connecting lesion to the mitral valve annulus; a bi-atrial procedure should be considered for those with persistent and longstanding persistent AF; when it can be safely performed, complete occlusion of the left atrial appendage should be considered.

Nowadays there are 3 innovative surgical procedures as stand-alone techniques that are currently performed [5]:

1. Unilateral epicardial thoracoscopic pulmonary vein isolation posterior encircling box lesion without left atrial appendage exclusion.

2. Alternative approaches to posterior left atrial wall epicardial ablation lesion without left atrial appendage exclusion.

3. Bilateral pulmonary vein isolation procedures inclusive of left atrial appendage exclusion: bilateral thoracoscopy or thoracotomy approach to perform isolated right and left Pulmonary Vein Antrum Isolation pairs with or without additional linear lesions on the left and right atrium. An alternative approach is to perform a posterior pulmonary vein isolation encircling box lesion.

The persistent activity of some active epicardial non isolated ganglionated plexi located on left atrial posterior wall, could explain the $10 \%$ to $40 \%$ recurrence rate of $\mathrm{AF}$ after surgical pulmonary veins isolation alone [6]. Combined pulmonary vein isolation (by endocardial approach) with left atrial posterior wall isolation and excision of left atrial appendage (by bilateral thoracoscopic epicardial approach) could lead to a success rate of $89 \%$ at 6 months [7].

\section{Posterior left atrial wall isolation in atrial fibrillation: rationale}

Electrical isolation of posterior left atrial wall in persistent AF aims to separate not only the four pulmonary vein considered as triggers but also the four ganglionated plexi of atrial myocardium causing an autonomic denervation. Ganglionated plexi is supposed to play significant role as an influencing factor for persistence of AF through a complex interconnection neural network. Epicardial ganglionated plexi include anterior right ganglionated plexus (ARGP), inferior right ganglionated plexus (IRGP), superior left ganglionated plexus (SLGP) and inferior left ganglionated plexus (ILGP). They are part of the intrinsic cardiac autonomic nervous system and could be identified on the left atrial epicardial wall in the vicinity of the pulmonary vein antrum (Figure 1) [3]. 


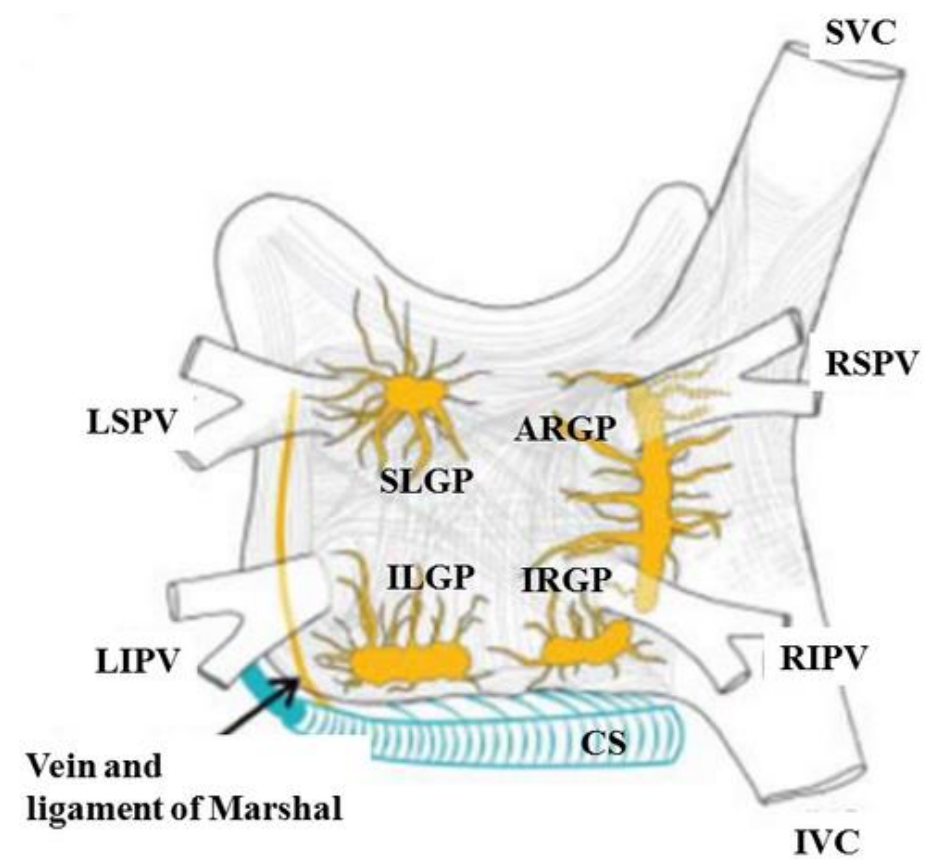

Fig. 1. Structures of left atrial posterior wall: ARGP - anterior right ganglionated plexus, CS - coronary sinus, IRGP inferior right ganglionated plexus, ILGP - inferior left ganglionated plexus, IVC - inferior vein cava, LSPV left superior pulmonaty vein, LIPV - left inferior pulmonary vein, RSPV - right superior pulmonary vein, RIPV right inferior pulmonary vein, SLGP - superior left ganglionated plexus; modified after [3].

The intrinsic cardiac autonomic nervous system may serve as a trigger or perpetuator for AF [8, 9]. Both components of autonomic nervous system (sympathetic and parasympathetic) are present in the ganglionated plexi embedded in the fat pads on the epicardial surface [10]. The ganglionated plexi represent an integration centre that modulates the autonomic innervation between extrinsic and intrinsic cardiac autonomic nervous system [11]. The parasympathetic activation induces widening of the window of atrial vulnerability while the sympathetic stimulation enhances automatic firing within the pulmonary vein, promoting atrial ectopic beats at the veno-atrial junction.

The response of ganglionated plexi stimulation is primarily vagal because the parasympathetic fibres are the major component of these structures [12]. Ganglionated plexi are identified by obtaining slowing of the ventricular rate in AF during endocardial or epicardial high frequency stimulation of specific sites of the left atrium [13]. The epicardial approach allows an easier identification of the major fat pads than with an endocardial one, due to direct visualization combined with visual oriented and electrical guided high frequency stimulation.

Vago-sympathetic denervation as a potential therapeutic modality for AF has been demonstrated first of all in animal models [1417]. Endocardial ablation of autonomic ganglia has been reported to be curative for AF [18]. Until now, no direct evidence exists of the completeness ganglionated plexi destruction or isolation during AF ablation in humans.

\section{Thoracoscopic epicardial posterior left atrial wall isolation: technique}

Epicardial right monolateral thoracoscopic approach using microwave or radiofrequency energy in patients with refractory persistent AF aims to create an electrical block around the posterior left atrial wall, isolating the pulmonary veins and ganglionated plexi, as an alternative to endocardial complex segmentation.

Symptomatic patients, aged $\geq 18$ years and documented refractory symptomatic persistent $A F$, referred for pulmonary vein isolation or patients who are not candidates for 
catheter ablation or who have failed to at least one attempt are considered eligible for this surgical procedure. Patients with advanced left ventricular systolic function impairment (ejection fraction <35\%), with significant chronic obstructive pulmonary disease (peak expiratory flow <40\%), comorbidity with reduced life expectancy, presence of left atrial or left atrial appendage thrombus (detected by transesophageal echocardiography) are not eligible for this stand-alone surgical procedure. However, the decision-making in these instances needs to be based on each institution's experience with catheter and surgical ablation of $\mathrm{AF}$, the relative outcomes and risks of each in the individual patient, and patient preference [3].

\section{Operative technique}

The procedure is performed under general anaesthesia and is divided into four major steps which include: patient positioning, ports placement, pericardial reflection dissection and positioning of the antenna followed by energy application [19]. The esophagus is easily identified by the surgeon after the transesophageal probe is introduced. The right vagus nerve is visualized in the mediastinum where it crossed the trachea and is tested before energy application, as a first step of the isolation procedure. The ARGP and IRGP are easily identified and stimulated under endoscopic visualization (Figure 2).

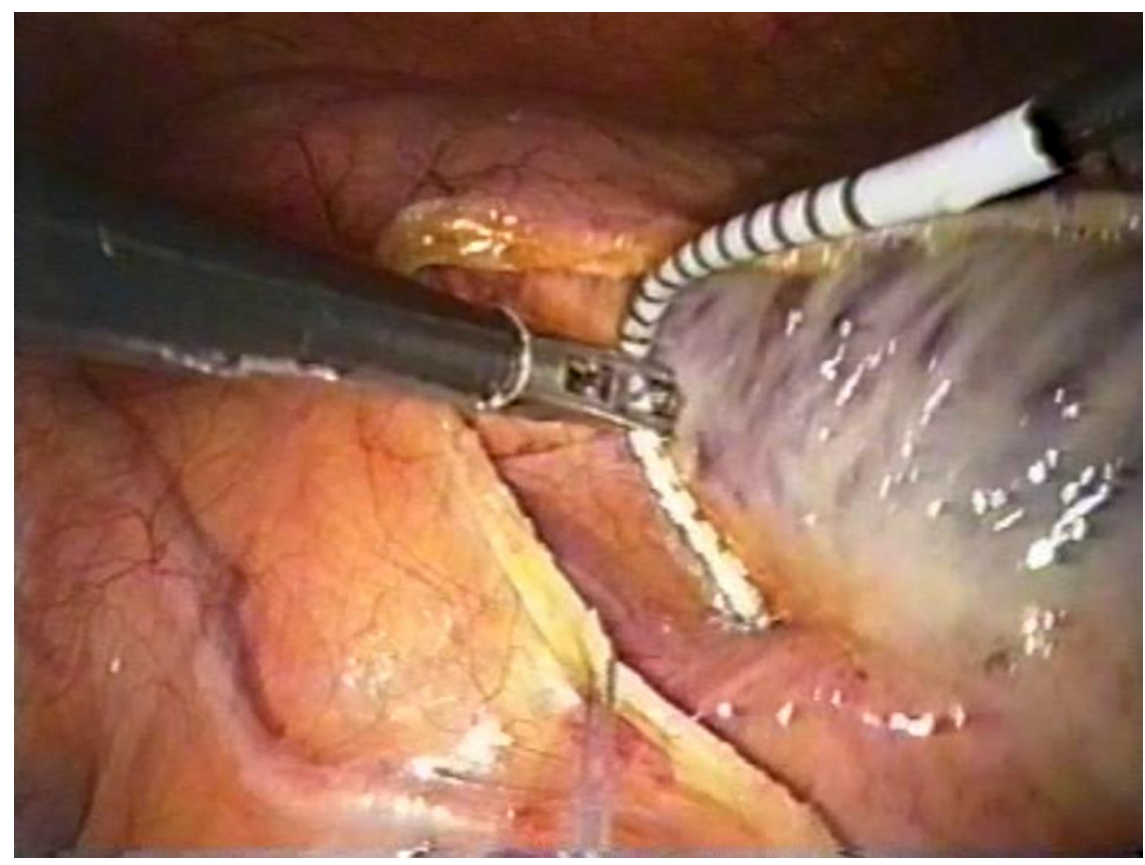

Fig. 2. Anatomical site of anterior right ganglionated plexus shown by duo-decapolar catheter (image during the isolation procedure). Courtesy of doctor Mark La Meir and Professor Luc De Roy (Belgium).

Access to the LSGP is obtained by introducing the stimulating electrode through the transverse sinus, starting at the superior vena cava and orientating towards to the aorta under endoscopic guidance. The entire larger area of the LSGP can be stimulated in a repeatable fashion by this technique. The ILGP could not be easily reached by this right sided approach. Epicardial mapping and testing of ganglionated plexi is performed by high frequency stimulation (with the following parameters: $20 \mathrm{~Hz}, 2 \mathrm{~ms}, 20 \mathrm{~mA}$, using a programmable cardiac stimulator) in the following sequence: ARGP, IRGP and finally, LSGP. Each vagal response of ganglionated plexi to high frequency stimulation is recorded before and after procedure and the results are compared to assess if ganglionated plexus activity was modified. Two sequential applications are performed to create a box lesion by a continuous encircling line around the 4 pulmonary veins. Two times encircling box lesion set is the procedure ablation endpoint. Each ablation segment is performed 
with a power setting of 65 watts for 120 seconds per lesion. After 2 complete encircling lesions, electrical isolation of the pulmonary vein is assessed by pacing the right pulmonary vein - left atrium junction inside the box lesion, when in sinus rhythm, verifying the absence of capture by the atrium. Entry and/or exit block across the box lines is confirmed by sensing and pacing with a duo-decapolar electrophysiological catheter in those patients who converted into sinus rhythm during the procedure. The ideal is to obtain a complete electrical block between the box lesion and the rest of left atrium myocardium.

\section{Pre and postablation care and follow-up}

Before the procedure, a 12 lead electrocardiogram recording, telemetry surveillance, respiratory function tests, 48 hours Holter monitoring, transthoracic and transesophageal echocardiography and computer-tomography of the pulmonary vein are performed in all patients. Antiarrhythmic medications except amiodarone are stopped more than five half-lives prior to ablation. All patients are taking acenocoumarol during 4-8 weeks before the intervention. Documented INR (International Normalized Ratio) between 2 and 3 , for at least 3 times is mandatory prior to the procedure. Acenocoumarol is replaced 3 days prior to the ablation by a therapeutic dose of low-molecular-weight heparin. Transoesophageal echocardiography is mandatory 24 hours prior to the intervention and during the procedure to rule out the presence of thrombus in the left atrium or left atrium appendage.

After the procedure the patients are monitored continuously for 24 hours in the intensive care unit. After leaving the intensive care unit antiarrhythmic medication (primarily amiodarone or sotalol) is reintroduced. Lowmolecular-weight heparin is restarted 24 hours after the procedure followed by an oral anticoagulant therapy (acenocoumarol) 72 hours later, after drainage tube is removed (usually after 24 hours). Patients are followed by continuous electrocardiograms and clinical monitoring during in-hospital stay.
The follow-up is planned at every 3 months and consisted in: clinical examination, 12 lead ECG, transthoracic echocardiography and 7 days Holter monitoring. Adverse events are evaluated during hospitalization and at every follow-up by the cardiologist. Antiarrhythmic and anticoagulation drugs are continued in all patients until the first follow-up.

\section{Thoracoscopic epicardial posterior left atrial wall isolation: preliminary results}

There has been over two decades of experience with stand-alone procedures. The first minimally invasive surgical approach using video-assisted pulmonary vein ablation and exclusion of the left atrial appendage was described twelve years ago in a patient with paroxysmal AF [20].

The success rate at six months follow-up after minimally invasive pulmonary vein isolation and partial autonomic denervation in patients with persistent or longstanding persistent AF was reported to be about $57 \%$ [21]. Another study with 114 patients published that $46.9 \%$ and $32 \%$ of patients with persistent and longstanding persistent AF were free of AF and off antiarrhythmic medications at 195 days of follow-up [22]. After thoracoscopic offpump radiofrequency pulmonary vein isolation plus left atrial appendage exclusion $60 \%$ to $80 \%$ of patients obtain sinus rhythm [23-26]. Moreover one systematic review suggested that the efficacy of stand-alone surgical ablation by bipolar radiofrequency is equivalent to the cut-and-sew maze III technique [4].

When connection lines to the mitral annulus are added the success rates seems to be comparable with the cut-and-sew Maze. There are three limitations to doing this on the full beating heart: visualization behind the full beating heart's left atrium is very limited; circumflex coronary artery overlying the mitral valve could be damage, and the coronary sinus is unreliable, and may leave a gap [4].

The efficacy and the safety of hybrid procedure which combine minim invasive epicardial right monolateral thoracoscopy with catheter endocardial ablation, in symptomatic refractory persistent AF was analysed in small 
studies [27-30]. It seems that a combined transvenous endocardial and thoracoscopic epicardial ablation procedure for $\mathrm{AF}$ is feasible and safe, with a single-procedure success rate of $83 \%$ at 1 year [28]. Although the true success rates of these procedures are likely to be lower than has been reported if more extensive monitoring will be performed in the future, these results are encouraging. Stable sinus rhythm in hybrid ablation of persistent AF results in important left atrial and left ventricle reverse remodelling after 12 months [31].

Hybrid AF ablation determines durable lesions and high rates of pulmonary vein isolation even after long-term follow-up. Most of the repeat procedures after the hybrid approach are related to left atrial flutters that could be successfully treated by catheter ablation [32]. The hybrid strategy (epicardial followed by endocardial catheter ablation) is feasible and safe with a high post-procedural success and seems to represent the optimal treatment with low risk load and potentially long-term benefit for patients with a persistent and long-standing persistent AF.

Prospective multicentre clinical trials are needed to better define the relative safety and efficacy of various surgical tools and techniques. There have been no randomized studies performed comparing the stand-alone

\section{References}

1. American College of Cardiology/American Heart Association Task Force on Practice Guidelines. 2014 AHA/ACC/HRS guideline for the management of patients with atrial fibrillation: a report of the American College of Cardiology/American Heart Association Task Force on Practice Guidelines and the Heart Rhythm Society. J Am Coll Cardiol 2014; 64(21):e1-76.

2. $A C C / A H A / E S C 2006$ Guidelines for the Management of Patients with Atrial Fibrillation. A Report of the American College of Cardiology/American Heart Association Task Force on Practice Guidelines and the European Society of Cardiology Committee for Practice Guidelines (Writing Committee to revise the 2001 Guidelines for the management of surgical treatment of AF with endocardial catheter ablation procedures.

This article could be a preliminary step of a future prospective study that aims to compare epicardial right monolateral thoracoscopy with endocardial catheter ablation in patients with persistent AF.

\section{Conclusion}

Minimally invasive epicardial right monolateral thoracoscopy (especially combined with catheter endocardial ablation) in symptomatic refractory persistent $A F$ is feasible and clinically effective. However, the efficacy of this approach on the long-term in curing refractory persistent AF remains to be demonstrated.

\section{Conflict of interest}

The author(s) declare that they have no competing interests.

\section{Funding}

This work was supported by the Project entitled: Expanding and upgrading an Atrial Fibrillation Treatment Research Center as a method of preventing heart failure by developing the research and development infrastructure.

patients with atrial fibrillation). Circulation 2006; 114:257-354.

3. 2012 HRS/EHRA/ECAS Expert Consensus Statement on Catheter and Surgical Ablation of Atrial Fibrillation: recommendations for patient selection, procedural techniques, patient management and follow-up, definitions, endpoints, and research trial design. Europace 2012; 14(4):528-606.

4. Huffman MD, Karmali KN, Berendsen MA, et al. Concomitant atrial fibrillation surgery for people undergoing cardiac surgery. Cochrane Database Syst Rev 2016; 22(8):CD011814.

5. Ad N, Damiano RJ Jr, Badhwar V, et al. Expert consensus guidelines: Examining surgical ablation for atrial fibrillation. $J$ Thorac Cardiovasc Surg 2017; 153(6):1330-1354. 
6. Misaki T, Fukahara K. Recent topics on the surgical treatment for atrial fibrillation. Ann Thorac Cardiovasc 2004; 10:277-280.

7. Blomstrom P, Nilsson L, Einarsson GM, Blomstrom-Lundqvist C. Video-assisted epicardial pulmonary vein isolation off-pump (VPVI) and ablation of ganglionic plexi in patients with atrial fibrillation. Our first 20 patients (Abstract). Heart Rhythm 2007(18); 1:128.

8. Horikawa-Tanami T, Hirao K, Furukawa $\mathrm{T}$, Isobe M. Mechanism of the conversion of a pulmonary vein tachycardia to atrial fibrillation in normal canine hearts: role of autonomic nerve stimulation. J Cardiovasc Electrophysiol 2007; 18(5):534-541.

9. Patterson E, Po SS, Scherlag BJ, Lazzara R. Triggered firing in pulmonary veins initiated by in vitro autonomic nerve stimulation. Heart Rhythm 2005; 2:624-631.

10. Verrier RL, Antzelevitch C. Autonomic aspects of arrhythmogenesis: the enduring and the new. Curr Opin Cardiol 2004; 19:2-11.

11. Hou Y, Scherlag BJ, Lin J, et al. Interactive atrial neural network: determining the connections between ganglionated plexi, Heart Rhythm 2007; 4:56-63.

12. Hou Y, Scherlag BJ, Lin J, et al. Ganglionated plexi modulate extrinsic cardiac autonomic nerve input effects on sinus rate, atrioventricular conduction, refractoriness, and inducibility of atrial fibrillation. J Am Coll Cardiol 2007; 50(1):61-68.

13. Nakagawa $H$, Scherlag BJ, Lockwood $D$, et al. Localisation of left atrial autonomic ganglionated plexuses using endocardial and epicardial high frequency stimulation in patients with atrial fibrillation. Heart Rhythm 2005; 2(5):S10-S11.

14. Randall WC, Ardell JL. Selective parasympathetectomy of autonomic and conductible tissues of the canine heart. $A m \mathrm{~J}$ Physiol 1985; 248:H61-H68.

15. Ardell JL, Randall WC. Selective vagal innervation of sinoatrial and atrio-ventricular nodes in canine heart. Am J Physiol 1986; 251:H764-H773.

16. Nakagawa H., Scherlog BJ, Aoyama H, et al. Catheter ablation of cardiac autonomic nerves for prevention of atrial fibrillation in a canine model (abstract). Heart Rhythm 2001; 1:S10.

17. Chevalier $P$, Obadia JF, Timour $Q$, et al. Thoracoscopic epicardial radiofrequency ablation for vagal atrial fibrillation in dogs. Pacing Clin Electrophysiol 1999; 22:880-886.

18. Scherlag GJ, Nakagawa M, Jackman WM, et al. Electrical stimulation to identify neural elements on the heart: their role in atrial fibrillation, J Interv Cardiovasc Electrophysiol 2005; 13:37-42.

19. La Meir M, De Roy L, Blommaert D, Buche M. Treatment of lone atrial fibrillation with a right thoracoscopic approach. Ann Thorac Surg 2007; 83:2244-2245.

20. Wolf RK, Schneeberger EW, Osterday R, et al. Video-assisted bilateral pulmonary vein isolation and left atrial appendage exclusion for atrial fibrillation. J Thorac Cardiovasc Surg 2005; 130(3):797-802.

21. Edgerton JR, Edgerton ZJ, Weaver $T$, et al. Minimally invasive pulmonary vein isolation and partial autonomic denervation for surgical treatment of atrial fibrillation. Ann Thorac Surg 2008; 86(1):35-38.

22. Edgerton JR, McClelland JH, Duke $D$, et al. Minimally invasive surgical ablation of atrial fibrillation: six-month results. $J$ Thorac Cardiovasc Surg 2009; 138(1):109-113.

23. De Maat GE, Pozzoli A, Scholten MF, et al. Surgical minimally invasive pulmonary vein isolation for lone atrial fibrillation. Innovations 2013; 8:410-415.

24. Doty JR, Clayson SE. Surgical treatment of isolated (lone) atrial fibrillation with Gemini-S ablation and left atrial appendage excision (GALAXY procedure). Innovations 2012; 7:3338.

25. McClelland JH, Duke D, Reddy R. Preliminary results of a limited thoracotomy: new approach to treat atrial fibrillation. $J$ Cardiovasc Electrophysiol 2007; 18:1289-1295.

26. Kasirajan V, Spradlin EA, Mormando TE, et al. Minimally invasive surgery using bipolar radiofrequency energy is effective treatment for refractory atrial fibrillation. Ann Thorac Surg 2012; 93:1456-1461.

27. La Meir M, Gelsomino S, Lorusso R, et al. The hybrid approach for the surgical treatment of lone atrial fibrillation: one-year results employing a monopolar radiofrequency source. J Cardiothorac Surg 2012; 7:71.

28. Pison L, La Meir M, van Opstal J, Blaauw Y, Maessen J, Crijins HJ. Hybrid thoracoscopic surgical and transvenous catheter ablation of atrial fibrillation. JACC 2012; 60:54-61.

29. La Meir M, Gelsomino $S$, Lucà $F$, et al. Minimally invasive surgical treatment of lone atrial fibrillation: Early results of hybrid versus standard minimally invasive approach employing radiofrequency sources. Int $J$ Cardiol 2013; 167:1469-1475.

30. Gelsomino S, Van Breugel HN, Pison L, et al. Hybrid thoracoscopic and transvenous catheter ablation of atrial fibrillation. Eur $J$ Cardiothorac Surg 2014; 45(3):401-407. 
31. Toplisek J, Pernat A, Ruzic N, et al. Improvement of atrial and ventricular remodeling with low atrial fibrillation burden after hybrid ablation of persistent atrial fibrillation. Pacing Clin Electrophysiol 2016; 39(3):216-224.
32. Velagic $V$, DE Asmundis $C$, Mugnai $G$, et al. Repeat procedures after hybrid thoracoscopic ablation in the setting of longstanding persistent atrial fibrillation: electrophysiological findings and 2-year clinical outcome. J Cardiovasc Electrophysiol 2016; 27(1):41-50. 